\title{
Lessons Learned from Research via Private Social Media Groups
}

\author{
K. Cassie Kresnye \\ Indiana University \\ ckresnye@iu.edu \\ Ben Jelen \\ Indiana University \\ bcjelen@indiana.edu
}

\section{Maria K. Wolters}

University of Edinburgh \& Alan Turing Inst.

maria.wolters@ed.ac.uk

\author{
Juan F. Maestre \\ Indiana University \\ jmaestre@indiana.edu
}

\author{
Mona Y. Alqassim \\ University of Edinburgh \\ mona.alqassim@ed.ac.uk
}

\section{Katie A. Siek}

Indiana University

ksiek@indiana.edu

\section{ABSTRACT}

As research methods evolve to provide a voice to understudied, distributed communities, we explore our experiences running and analyzing Asynchronous Remote Communities (ARC) studies. Our experiences stem from four separate Facebook-based ARC studies with people who experience: rare disease, pregnancy, miscarriage, or HIV. We delve into these studies' methods, and present updated guidelines focused on improved study design, data collection, and analysis plans for ARC studies.

\section{CCS CONCEPTS}

- Human-centered computing $\rightarrow$ Web-based interaction; User studies;

Permission to make digital or hard copies of part or all of this work for personal or classroom use is granted without fee provided that copies are not made or distributed for profit or commercial advantage and that copies bear this notice and the full citation on the first page. Copyrights for third-party components of this work must be honored. For all other uses, contact the owner/author(s).

CHI'19 Extended Abstracts, May 4-9 2019, Glasgow, Scotland UK

(c) 2019 Copyright held by the owner/author(s).

ACM ISBN 978-1-4503-5971-9/19/05.

https://doi.org/10.1145/3290607.3299055 
Table 1: Summary of 4 ARC Studies the Authors Facilitated

\begin{tabular}{lccccc} 
& N & Grps & Wks & Actv & Int'I \\
\hline Rare[1] & 13 & 1 & 22 & 11 & Yes \\
HIV[5] & 19 & 1 & 8 & 10 & Yes \\
M/C & 42 & 2 & 8 & 16 & Yes \\
Preg & 20 & 3 & 16 & 32 & No \\
\hline
\end{tabular}

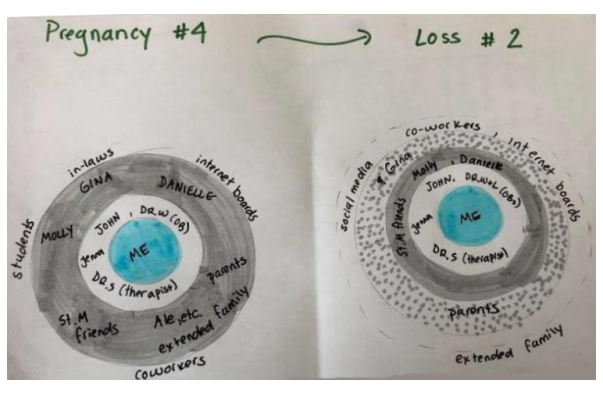

Figure 1: Example of a media response showing how support networks change pre and post miscarriage. This is a 'media' activity that participants must upload, and may result in lower participation due to those extra steps.

\section{KEYWORDS}

Asynchronous Remote Communities; Facebook; social media; research methods

\section{ACM Reference format:}

K. Cassie Kresnye, Juan F. Maestre, Ben Jelen, Mona Y. Alqassim, Maria K. Wolters, and Katie A. Siek. 2019. Lessons Learned from Research via Private Social Media Groups. In Proceedings of May 4-9, 2019, Glasgow, Scotland UK, CHI Conference on Human Factors in Computing Systems Extended Abstracts (CHI '19 Extended Abstracts), May 4-9 2019, Glasgow, Scotland UK, (CHI'19 Extended Abstracts), 8 pages. ACM, New York, NY. https://doi.org/10.1145/3290607.3299055

\section{INTRODUCTION}

Researchers are developing new methods to utilize evolving social media streams to engage understudied groups. The Asynchronous Remote Communities (ARC) method [3] is one of the emerging methods in $\mathrm{HCl}$ for leveraging social media benefits which has been extended and evaluated with multiple populations [1,5-7]. As with any method, there are implicit assumptions and unseen hurdles In this paper, we explicitly discuss ARC issues related to facilitation and data analysis and contribute six new lessons for the $\mathrm{CHI}$ community to utilize.

\section{METHOD}

Asynchronous Remote Community (ARC) is a recent method developed by MacLeod et al. [3]. ARC employs a social media platform to conduct research thereby gaining a better understanding of the needs and scope of solutions for distributed and typically understudied communities. ARC is especially beneficial to engage with study populations that are hard-to-access or have availability constraints. MacLeod et al. [2] conducted a group-based needs assessment study using ARC with rare disease populations. Researchers utilized ARC to understand the needs of pregnant women and new mothers [7] and individuals with HIV [5]. MacLeod et al. [2], Prabhakar et al. [7], and Maestre et al [5] encourage the $\mathrm{HCl}$ community to adapt this method and report on their methodological findings.

We describe our experiences using ARC over four studies shown in Table 1 that vary in number of participants (N); how many ARC groups facilitated (Grps); the study length in weeks (Wks); how many activities administered (Actv); and if there were international participants (Int'l). Our studies included four communities: rare disease [1] (Rare), HIV [5], miscarriage support (M/C) (unpublished), and pregnancy experiences (Preg) (unpublished). All studies were ethics board approved.

Consent Process. In Rare and HIV, participants printed the consent form and emailed a signed copy to researchers. In $\mathrm{M} / \mathrm{C}$ and Preg, participants were emailed a consent document and replied with a statement indicating consent. Since the M/C study had international collaborators, each research team consented from their own country. 


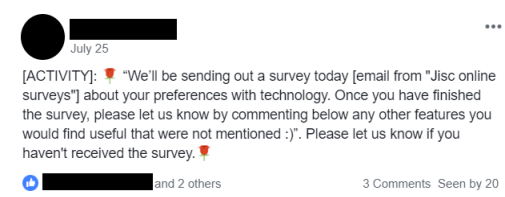

Figure 2: A notification to participants that a survey was emailed to them.

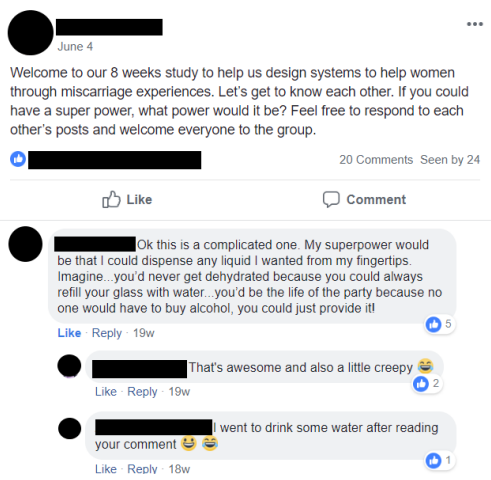

Figure 3: An example discussion post, this particular one being an icebreaker activity. These activities work best if limited to one or two questions.

\section{Study Procedures}

Each of these studies used a similar set of activities modified from $\mathrm{HCl}$ methods distributed equally over the study duration. These activities included media activities (e.g., uploading a photo shown in Figure 1), surveys (Figure 2), and free response discussions (Figure 3). These results were analyzed using qualitative methods such as open coding[8].

\section{FINDINGS AND DISCUSSION ON PREVIOUS LESSONS}

The presented lessons, summarized in Table 2, emerged from studies using varied populations. We categorize these lessons based on when in the research process they are most relevant and provide context via short examples.

\section{Recruitment Lessons}

The most successful method of recruitment was through existing online communities, specifically Facebook for our studies. Thus, it is vital to work with current community administrators to access these populations, as outlined in lesson 10 [4] and 18 [5] from Table 2. We found participants from established groups had higher social media literacy awareness and familiarity with online discussions During recruitment, a researcher went to where participants currently interacted and contacted a group administrator using their personal accounts (lesson 20 [5]) with ethics board approved study materials. We then asked permission to either join the group for recruitment purposes or ask the administrator to post on our behalf to build rapport (lesson 1 [4]).

\section{Activity Design Lessons}

Understanding the context in which people take part in the study ensures participants have the means and time to complete the activities - especially for participants with limited availability due to health or safety considerations (e.g., having a newborn). We emailed or messaged (lessons 3 and 4 [4]) individual participants to prompt activity completion. Sequential activities were possible, but only if adequate time was given between activities (Lesson 8). For example, M/C and Preg used 'problem and solution' activities, where participants discussed problems they faced in one activity and then discussed solutions to the top 3 problems in an activity two weeks later. This spacing aided participation rates and confirmed that lesson 8 [4] is not a hard rule but a reminder to be mindful of participants schedules.

\section{Group Interactions Lessons}

The remaining lessons set groundwork for efficiently running these online groups. Quickly responding to questions (lesson 22 [5]) was important, especially in M/C, HIV, and Rare due to international 
Table 2: ARC lessons MacLeod et al. [1-11] Prabhakar et al. [12-17] Maestre et al. [18-22]. For Category, we grouped lessons based on Recruitment (R), Activity Design (A), Group Interaction (G), Data Analysis (D) respectively.

Lessons

1 Building a strong rapport with members of groups used for recruiting before, during, and after the study

2 Investigating alternative methods of consenting electronically

3 Encouraging participants to provide feedback and build on each other's creative contributions.

4 Encouraging participants to post directly to the group, even submissions still in progress.

5 Exercising caution when planning the study and selecting activities.

6 Taking time to understand potential participant's Facebook behavior to inform decisions about when and how often to post and the overall study duration.

7 Researchers should be prepared to adopt multiple approaches to ensure activities are seen by participants.

8 Discouraging activities that build on one another where sequence is important.

9 Providing opportunities for socialization between participants that are separate from formal study activities.

10 Giving careful consideration to the makeup of the group when recruiting, targeting either a highly homogeneous population or targeting several homogeneous groups.

11 Being mindful of the number of input mechanisms. Each additional input mechanism introduces additional overhead.

12 Being mindful of the number of steps needed to complete an activity when selecting activities for the ARC method.

13 Consideration of participant's technology preferences, while developing activities.

14 Find balance between the ease of use and the usefulness of the data collection activities while engaging participants.

15 Adapt commonly used data capturing mechanisms used in FtF focus groups to use in online, asynchronous research methods.

16 Careful consideration of activity selection. When possible, we must provide meaningful and helpful interventions

17 Researchers should be proactive about using strategies to make data collection and organization easier.

18 Make support group administrators understand, approve, and be part of the study so that they can vouch for the intention, safety, and potential benefits of the study.

19 Post activities and reminders on the same days every week.

20 Researchers should use their personal accounts when studying stigmatized populations with the ARC method on a social media platform like Facebook.

21 Learn about the type of language to use when communicating with the stigmatized before using ARC.

22 Be prepared to clarify miscommunication and answer questions as soon as possible to avoid unnecessary distractions and misunderstandings.

23 Carefully weigh the pros and cons of potential platforms.

24 Create a detailed outline of data collection plans to ensure correct data is captured.

25 Consistently collect and store data during the course of the study.

26 Meet with research team frequently to reflect on activities.

27 Create a living team timeline and iterate on it over the course of the study.

28 Foster the group relationship after study completion.

Category

$\mathrm{R}$

R

A

A

A

A

A

A

G

R

A

A

A

A

A

A

D

$\mathrm{R}$

A

R

R

G




\section{Sidebar 1: Platform Selection Criteria}

\section{Platform Selection Considerations}

- Ease of individual contribution

- Interaction methods

- Navigation between activities

- Research legality

- Platform privacy policies

- Data lifetime - for researchers (e.g., time stamp accuracy) and participants

- Population platform literacy

- Data collection options (e.g., API, manual)

- Current online meeting place for participants

- Platform concepts

- Costs (for platform or resources to teach participants a new platform)

- Recruitment Potential (e.g., snowball sampling via sharing posts; advertisements) participants. Researchers should work out a schedule of monitoring the groups they are running, ensuring a safe environment for participant interactions. Many platforms allow for direct notifications when participants are active that can help the researcher who is currently on-call to moderate and collect data. Understanding the population being studied (lesson 21 [5]) is another key to a successful ARC study. While this is important for any participant studies, it is a vital step in ARC studies because the communication is only through written means. This forces nuances, such as body language and inflection, to be inferred. Researchers should be aware of communities' online interaction conventions, such as acronyms, jargon, and memes that a researcher must understand not only when analyzing the data but to moderate the group fairly.

\section{FINDINGS AND DISCUSSION OF NEW LESSONS}

Reflecting on the prior lessons, we realized platform selection, data collection, and analysis of an ARC study were not well discussed. We share insights we gained as we overcame many of the hurdles we faced in each of these areas.

\section{Choosing a Platform}

Choosing a platform (lesson 23) is a key first step to developing an ARC study. Our studies used Facebook because that was where our participants were already talking to each other about the topic of the study (rare disease, HIV, miscarriage, pregnancy), but ARC has potential to be explored in other online community platforms too. Benefits of Facebook included its large international user base, built-in private groups, and the ability to post photos. Key challenges included: (1) adding participants to a private group because the researcher and participants must already be connected as 'Facebook Friends,' which typically presupposes a connection beyond the research; (2) extracting the data because of API limitations and data lifetime limits (e.g., timestamps change from minutes, hours, days, weeks ago); and (3) Facebook's privacy issues are well documented.

Researchers should prioritize a platform that will help them answer their research question while balancing the considerations outlined in Sidebar 1. The platform should provide participants the ability to easily join the group, contribute to discussions, and navigate activities. Researchers should consider any restrictions a platform may have on activities (e.g., multi-question survey mechanisms) and how data is collected - which can be laborious if collection is manual. Researchers need to be aware of the rules and regulations that govern the use of each platform. This ranges from platform-specific conventions about use for research purposes to the rules for storing, managing, and exporting data that research participants generate on the platform.

Understanding Platform Concepts. Some social media platforms have features to understand group interactions, but these can be less useful than they appear. For example, when an individual 'sees' a 


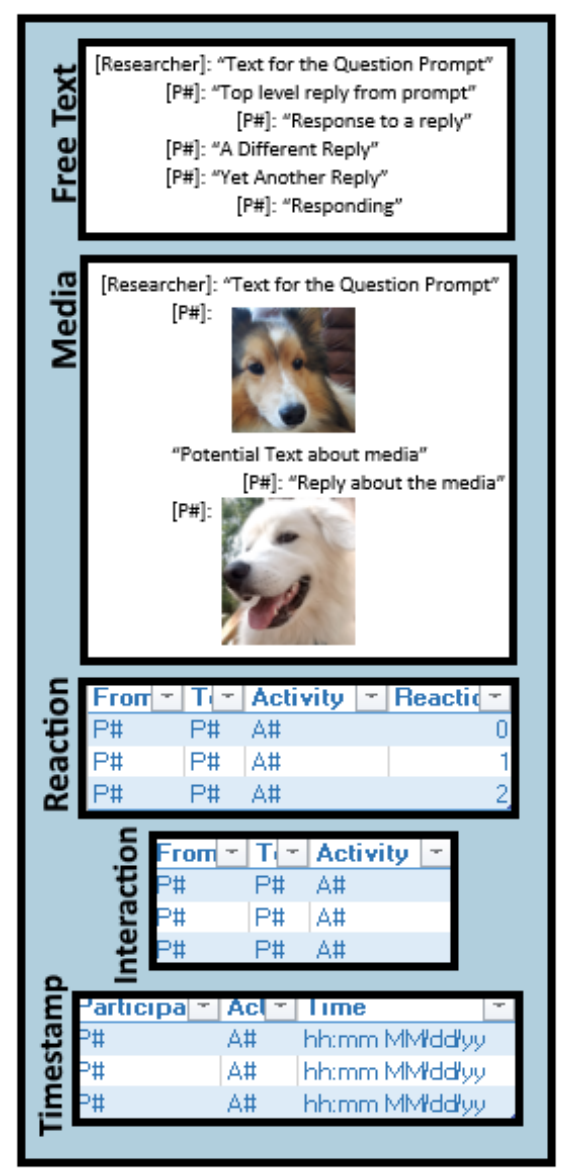

Figure 4: Example of an outline for storing data from Facebook Groups. The spacing indications allow for an easier qualitative coding. Facebook reactions and interactions were also tracked. Timestamp data corresponds to participants' first comment on the activity. post on Facebook, this means only that the post appeared on their dashboard or on the group page, but not that they actively engaged with it. Researchers should keep this in mind during data analysis.

\section{Detailing a Data Collection Outline}

ARC studies have the ability to produce vast amounts of data. With this in mind, our second additional lesson (lesson 24) is to have a detailed data collection outline. The outline should account for what specific data should be collected and what format it should be stored in such as in Figure 4. The collection plan should also include what data should be anonymous before storing (e.g., participant names) and roughly outline study personnel responsibilities. Lastly, the research team can use the plan to include protocols for dealing with missing data. ARC participation is often high, but may still result in participants missing a few activities or participating later.

The Challenge with Time Zones. Our studies involved individuals in different time zones that created challenges regarding analyzing when individuals interacted with the materials. Specific rules should be established from the beginning of the study about when researchers will post activities for the best chance of engagement with all participants and how time stamp data will be analyzed. In $M / C$ and Preg, we used 'day' units, which were defined as 24 hours after an activity was published. If an activity was published at 9am, then we counted any participation before 9am the next day as participation within 1 day.

Data Collection Schedule. Consistency for the participants in the study is important (lesson 19), however we also found consistency important for researchers during data collection (lesson 25). The platform used for our studies had established hurdles for automatic data collection, thus we manually exported the data from the groups. In M/C and Preg, we implemented a weekly export that was divided between researchers to distribute the workload. Researchers stored the new activity data and updated previous activity data if there were any changes (e.g., a participant responding late).

Such a consistent schedule, coordinated between researchers, is best set up in coordination with the activity plan and allows for a smoother transition to data analysis. It also promotes ongoing reflection on the data being collected and makes it easier to post pertinent follow up activities while the issues that prompted them are still fresh in the participants' minds. Researchers can use a variety of analysis techniques. Current ARC studies have used open coding, affinity diagramming.

\section{Meeting with the Research Team Frequently}

The study team should also strive to meet weekly during the study (lesson 26) to get updates on activity participation and assess how activities are fulfilling research questions. Check-ins are especially useful if activities that require a certain order are used. A meeting can evaluate if the group is ready for the upcoming activity or push it to the next week to allow for more response time. During meetings, we 


\section{Table 3: Activity Descriptors Info}

Type of Activity
Targeted RQs
Triangulation between Activities
Posting Researcher
Publishing Date
Data Generation
Ordering Rules
Preparation Required

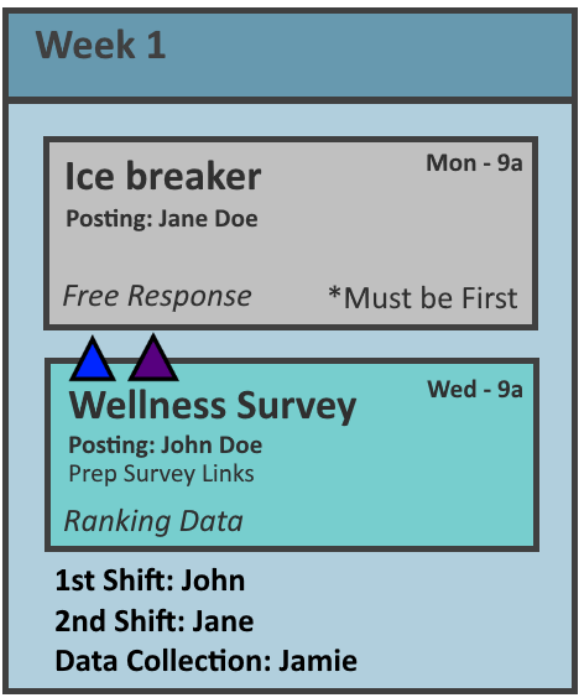

Figure 5: Example Module for creating a ARC study timeline. The purple and blue triangles indicate triangulation with other activities based on color. Numbers can be used as well. often discussed new activities based on the findings of previous responses. New activities ranged from a small survey to rank different discussion points from the previous week or an advice column prompt to delve more into a single topic. We set aside time each week to discuss a strong debrief activity that includes elements discussed throughout the study.

\section{Crafting a Living Timeline}

Flexibility when planning these studies works best if paired with a shared, living timeline for all members of the research team (lesson 27). This timeline should include details regarding activity preparations and data collection plans with assigned researchers to designated times. Table 3 outlines information that should be included for each activity on the timeline. Activity modules may be used for this purpose, allowing for quick communication of information as well as interchangeability.

Activity Module. Each activity module should be movable, as changes to the study may happen, while also including information on weekly responsibilities. Researchers should discuss how activity module shifts impact moderating and data collection responsibilities. We used a digital form for $\mathrm{M} / \mathrm{C}$ and Preg that inspired our proposed design in Figure 5. Researchers could use a physical form for hands-on collaboration, or it could be ported to specialized project management tools, such as Trello.

\section{Growing a Participant Pool}

ARC provides a unique opportunity for a continued relationship with a participant group. Growing this relationship over the course of the study can result in a strong rapport and opportunities to continue research with the pool, thus it is important to balance this opportunity and allow participants freedom to engage as they see fit. In our studies, we had the last activity outline that the study is concluded and reminded them that they are able to leave the group, however we would post research updates and publication pre-prints for feedback in the private group to foster relationships (lesson 28).

\section{Engaging Diverse Groups}

Even though Facebook is the largest social media platform in the world at the time of writing, participants were often mostly white and relatively highly educated. Despite efforts to recruit widely - from diverse researchers (the $\mathrm{M} / \mathrm{C}$ research team had three women of color) to Facebook ads with women of color (Preg), - few participants from minority groups responded to study calls. Even when underrepresented groups enrolled in the study, they may not interact much within the ARC group. We observed this in M/C, where several Arab Muslim women enrolled, however most only participated in questionnaire activities. The need to keep ARC groups large enough to allow for continued interaction also meant that some group members may not feel comfortable disclosing personal information within the group. 


\section{CONCLUSION AND FUTURE WORK}

The existing ARC literature $[4,5,7]$ provides a great base to understand how to recruit, design activities, and foster group interactions, however as we reflected on the four ARC studies we have facilitated, we realized the research community would benefit from learning more about platform selection considerations, data collection plans, and how to dynamically modify activities based on ARC group participation and data generation. We also want to highlight the challenges of engaging diverse populations with ARC. We look forward to exploring ARC on additional social media platforms (e.g., Slack) and how the lessons learned need to be adapted since platforms have different user profiles, interaction patterns, privacy policies, and options for handling multi-modal data.

\section{ACKNOWLEDGMENTS}

We thank the study participants and Augustus. We were supported in part by the National Science Foundation (IIS-1560276, CNS-1560276), Indiana University Precision Health, and Ministry of Higher Education (Government of the Kingdom of Saudi Arabia), the Alan Turing Institute (EPSRC, EP/N510129/1).

\section{REFERENCES}

[1] Haley MacLeod, Grace Bastin, Leslie S Liu, Katie Siek, and Kay Connelly. 2017. Be Grateful You Don't Have a Real Disease: Understanding Rare Disease Relationships. In CHI 2017. ACM, 1660-1673.

[2] Haley MacLeod, Ben Jelen, Annu Prabhakar, Lora Oehlberg, Katie Siek, and Kay Connelly. 2016. Asynchronous Remote Communities (ARC) for Researching Distributed Populations. In 10th EAI International Conference on Pervasive Computing. ICST, 1-8. http://dl.acm.org/citation.cfm?id=3021319.3021320

[3] Haley MacLeod, Ben Jelen, Annu Prabhakar, Lora Oehlberg, Katie Siek, and Kay Connelly. 2017. A Guide to Using Asynchronous Remote Communities (ARC) for Researching Distributed Populations. EAl Endorsed Transactions on Pervasive Health and Technology 3, 11 (7 2017). https://doi.org/10.4108/eai.18-7-2017.152898

[4] Haley MacLeod, Ben Jelen, Annu Prabhakar, Lora Oehlberg, Katie A Siek, and Kay Connelly. 2016. Lessons learned from conducting group-based research on facebook. In CHI 2016. ACM, 804-815.

[5] Juan F Maestre, Haley MacLeod, Ciabhan L Connelly, Julia C Dunbar, Jordan Beck, Katie A Siek, and Patrick C Shih. 2018. Defining through expansion: conducting asynchronous remote communities (ARC) research with stigmatized groups. In CHI 2018. ACM, 557.

[6] Annu Sible Prabhakar, Lucia Guerra-Reyes, Anne Effron, Vanessa M Kleinschmidt, Maggie Driscoll, Charles Peters, Vanessa Pereira, Majdah Alshehri, Tom Ongwere, and Katie A Siek. 2017. Let me know if you need anything: Support realities of new mothers. In 11th EAI International Conference on Pervasive Computing. ACM, 31-40.

[7] Annu Sible Prabhakar, Lucia Guerra-Reyes, Vanessa M Kleinschmidt, Ben Jelen, Haley MacLeod, Kay Connelly, and Katie A Siek. 2017. Investigating the suitability of the asynchronous, remote, community-based method for pregnant and new mothers. In CHI 2017. ACM, 4924-4934.

[8] Anselm Strauss and Juliet Corbin. 1990. Basics of qualitative research. Sage publications. 